\title{
Genome subtraction for the identification of potential antimicrobial targets in Xanthomonas oryzae pv. oryzae PXO99A pathogenic to rice
}

\author{
V. Keshri • Dhananjaya P. Singh $\cdot$ R. Prabha $\cdot$ \\ A. Rai · A. K. Sharma
}

Received: 10 January 2013/Accepted: 19 March 2013/Published online: 4 April 2013

(C) The Author(s) 2013. This article is published with open access at Springerlink.com

\begin{abstract}
In pathogenic bacteria, identification of essential proteins which are non-homologous to the host plants represents potential antimicrobial targets. We applied subtractive genomics approach for the identification of novel antimicrobial targets in Xanthomonas oryzae pv. oryzae PXO99A, the causative agent of bacterial blight in rice. Comparative analysis was performed through BLAST available with the NCBI. The analysis revealed that 27 essential protein sequences out of 4,988 sequences of $X$. oryzae pv. oryzae PXO99A are non-homologous to Oryza sativa. Subsequent analysis of 27 essential proteins revealed their involvement in different metabolic activities such as transport activity, DNA binding, structural constituent of ribosome, cell division, translation, and plasma membrane. These 27 proteins were analyzed for virulence and novelty and out of 27 , three essential non-homologous proteins were found to be the novel antimicrobial targets.
\end{abstract}

Keywords Xanthomonas oryzae - Rice - Pathogen · Essential genes - Subtractive genomics

V. Keshri · D. P. Singh $(\bowtie) \cdot$ R. Prabha · A. K. Sharma National Bureau of Agriculturally Important Microorganisms, Indian Council of Agricultural Research, Kushmaur, Maunath Bhanjan 275101, UP, India

e-mail: dpsfarm@rediffmail.com

\section{A. Rai}

Indian Agricultural Statistical Research Institute, Indian Council of Agricultural Research, Library Avenue, Pusa, New Delhi 110012, India

\section{Introduction}

Generation of vast genomic data from prokaryotic whole genome projects in the recent years has opened new avenues for finding out novel drug targets in microbes (Buysse 2001). Genome sequences of pathogenic microbes provided tremendous information which is now facilitating in silico identification and characterization of potential therapeutic targets and virulence factors of pathogens (Amineni et al. 2010; Dutta et al. 2006; Miesel et al. 2003). Potential therapeutic targets should be an essential component of a particular metabolic pathway in a pathogen, should be adequately selective to yield a drug that is specific against the pathogen and should possess no homolog within the host system so that the designed lead molecule can act against the functionality of the pathogen only but not against the host. Subtractive genomics approach entwined with bioinformatics can find out optimal targets related to previously unknown cellular functions in microbes based on the understanding of relatively similar biological processes in pathogens and hosts (Vetrivel et al. 2011; Koteswara et al. 2010; Sakharkar et al. 2004). Using this approach, a number of potential drug targets have been identified for bacterial pathogens of humans (Barh et al. 2011).

Search for antibacterial targets in bacteria pathogenic to plants has remained an untouched area of in silico research, although there exists tremendous scope for likely work with the appearance of a large volume of data sets coming out from the whole genome sequencing projects of the phytopathogenic bacteria. Xanthomonas oryzae pv. oryzae, a gamma-proteobacteria is an important pathogen of rice (Swings et al. 1990) causing bacterial leaf blight (Niño-Liu et al. 2006) or bacterial blight (Salzberg et al. 2008). Highyielding rice cultivars are more susceptible to the disease that leads to wilting of seedlings, yellowing and drying of 
leaves and yield loss. Besides physical disease management practices including sanitation, seed treatment with bleaching powder $(10 \mu \mathrm{g} / \mathrm{ml})$ and zinc sulfate $(2 \%)$ are reported to reduce the disease but chemical control using antibiotics has only limited success (Rice Knowledge Bank 2009). Continuously increasing resistance among the pathogens towards antibiotics has generated the need for searching

Table 1 Genomic data (NCBI 2012) of Xanthomonas oryzae pv. oryzae PXO99A

\begin{tabular}{|c|c|}
\hline Organism & $\begin{array}{l}\text { Xanthomonas oryzae pv } \\
\text { oryzae PXO99A }\end{array}$ \\
\hline Taxonomy ID & 360094 \\
\hline Chromosome & Single circular \\
\hline Protein & 4,988 \\
\hline Protein coding genes & 5,083 \\
\hline $\begin{array}{l}\text { Protein coding genes not found in other } \\
\text { strains (KACC10331 or MAFF311018) }\end{array}$ & 87 \\
\hline Genome size & $5,240,075 \mathrm{bp}$ \\
\hline GC \% & 63.6 \\
\hline
\end{tabular}

novel antimicrobial targets in pathogenic bacteria that may lead to the development of novel antimicrobial agents.

Xanthomonas oryzae pv. oryzae strain PXO99A is virulent towards many rice varieties representing diverse genetic sources for resistance and needs novel antimicrobials for reducing leaf blight resistance and increasing rice yield. Complete genome sequences of different strains of X. oryzae pv. oryzae like PXO99A (Salzberg et al. 2008), KACC10331, and MAFF311018 (Triplett et al. 2011) facilitated in-depth comparative genomic analyses. Genome comparison indicated that strain PXO99A contains various virulence-associated transcription activator-like effector genes and possesses a minimum of 10 major chromosomal rearrangements in comparison to the other strains KACC10331 and MAFF311018 (Salzberg et al. 2008). Looking into the practical implications of such work, we applied subtractive genomics approach to identify novel protein targets that encode pathogenicity in $X$. oryzae pv. oryzae PXO99A and help in finding out novel antimicrobial targets to develop potential antimicrobial agents against this important disease of rice.
Table 2 Most probable antibacterial targets in Xanthomonas oryzae pv. Oryzae

\begin{tabular}{|c|c|}
\hline GI/accession number & Description \\
\hline gil188574599|ref|YP_001911528.1| & TetR family transcriptional regulator \\
\hline gil188574639|ref|YP_001911568.1| & Twin arginine-targeting protein translocase TatC \\
\hline gil188574650|ref|YP_001911579.1| & TonB-dependent receptor \\
\hline gil188574873|ref|YP_001911802.1| & Isocitrate dehydrogenase \\
\hline gil188575043|ref|YP_001911972.1l & Monovalent cation $/ \mathrm{H}+$ antiporter subunit A \\
\hline gil188575296|ref|YP_001912225.1l & Cell division protein FtsW \\
\hline gil188575690|ref|YP_001912619.1l & Signal protein with PAS(PAC), GGDEF and EAL domains \\
\hline gil188575924|ref|YP_001912853.1। & Carbon storage regulator \\
\hline gil188576015|ref|YP_001912944.1| & Chemotaxis signal transduction protein \\
\hline gil188576081|ref|YP_001913010.1। & AcrB protein \\
\hline gil188576225|ref|YP_001913154.1| & Argininosuccinate lyase \\
\hline gil188576521|ref|YP_001913450.1l & Transposase \\
\hline gil188577176|ref|YP_001914105.1| & Lipid-A-disaccharide synthase \\
\hline gil188577531|ref|YP_001914460.1| & TonB-dependent outer membrane receptor \\
\hline gil188577685|ref|YP_001914614.1| & NADH dehydrogenase subunit $\mathrm{L}$ \\
\hline gil188577690|ref|YP_001914619.1| & NADH dehydrogenase subunit $\mathrm{G}$ \\
\hline gil188577693|ref|YP_001914622.1l & NADH dehydrogenase subunit D \\
\hline gil188577770|ref|YP_001914699.1| & Protein TldD \\
\hline gil188578034|ref|YP_001914963.1l & TonB-dependent outer membrane Receptor \\
\hline gil188578050|ref|YP_001914979.1l & cob(I)alamin adenosyltransferase \\
\hline gil188578410|ref|YP_001915339.1| & Sulfate $\mathrm{ABC}$ transporter permease \\
\hline gil188578579|ref|YP_001915508.1| & TonB-dependent outer membrane receptor \\
\hline gil188578592|ref|YP_001915521.1l & General secretion pathway protein $F$ \\
\hline gil188578888|ref|YP_001915817.1l & Chromosome partitioning protein \\
\hline gil188579158|ref|YP_001916087.1। & Xylosidase \\
\hline gil188579258|ref|YP_001916187.1l & 50S ribosomal protein L34 \\
\hline gi|229358035|ref|YP_001915183.2l & 50S ribosomal protein L31 \\
\hline
\end{tabular}




\section{Results and discussion}

Computational approaches have been applied to identify essential genes in prokaryotes. We reported the identification of essential genes as the potential antibacterial targets in plant pathogenic bacteria $X$. oryzae pv. oryzae. The approach was based on sequence alignment of proteins downloaded from the NCBI (Table 1) and database of essential genes (DEG). X. oryzae pv. oryzae PXO99A, O. sativa and database of essential genes (DEG) of prokaryotes contain 4,988, 21,342 and 7,643 protein sequences, respectively. Our results revealed that out of 4,988 proteins in X.oryzae pv. oryzae PXO99A, 406 unique sequences did not resulted in any hits (no hits found) and did not align with any sequence of $O$. sativa. The result is in agreement with the earlier reports (Jacobs et al. 2003, Sakharkar et al. 2004) who reported classified 300-400 essential genes in another bacteria $P$. aeruginosa. When non-homologous 406 sequences were aligned (two-way BLAST) against prokaryotic essential protein sequences of DEG with an e-value cutoff of $10^{-10}$ for determination of their essentiality, 27 sequences were found essential for the pathogen. Further functional categorization based on the respective gene description or name of these proteins revealed that in the pathogen, these proteins might be considered as unique and linked with the essential metabolic pathway. All these 27 protein sequences were related to different functional cellular properties such as transport activity, DNA binding, structural constituent of ribosome, cell division, translation, plasma membrane and membrane protein (Table 2).

The KEGG GENES database which is a resource for cross-species annotation of all available genomes by KEGG orthology (KO) system, classified all 27 essential genes of $X$. oryzae pv. oryzae into different categories according to their involvement in different metabolic pathways (Table 3). Metabolic pathway analysis of essential proteins revealed that majorly three genes are involved in oxidative phosphorylation, three in nitrogen metabolism, two in bacterial secretion system, one in glutathione metabolism, one in arginine and proline metabolism and

Table 3 KEGG orthology of all essential potential antimicrobial targets

\begin{tabular}{|c|c|c|}
\hline GI/accession number & $\mathrm{KO}$ & KO description \\
\hline gil188574599|ref|YP_001911528.1| & - & - \\
\hline gil188574639|ref|YP_001911568.1| & K03118 & Sec-independent protein translocase protein TatC \\
\hline gil188574650|ref|YP_001911579.1। & - & - \\
\hline gil188574873|ref|YP_001911802.1। & K00031 & Isocitrate dehydrogenase \\
\hline gil188575043|ref|YP_001911972.1। & K05559 & Multicomponent $\mathrm{K}+: \mathrm{H}+$ antiporter subunit $\mathrm{A}$ \\
\hline gil188575296|ref|YP_001912225.1। & K03588 & Cell division protein FtsW \\
\hline gil188575690|reflYP_001912619.1। & - & - \\
\hline gil188575924|ref|YP_001912853.1। & K03563 & Carbon storage regulator \\
\hline gil188576015|reflYP_001912944.1| & K03408 & Purine-binding chemotaxis protein $\mathrm{CheW}$ \\
\hline gil188576081|ref|YP_001913010.1। & K03296 & Hydrophobic/amphiphilic exporter-1 (mainly G-bacteria), HAE1 family \\
\hline gil188576225|reflYP_001913154.1| & K01755 & Argininosuccinate lyase \\
\hline gil188576521|ref|YP_001913450.1| & - & - \\
\hline gil188577176|reflYP_001914105.1। & K00748 & Lipid-A-disaccharide synthase \\
\hline gil188577531|ref|YP_001914460.1। & - & - \\
\hline gil188577685|ref|YP_001914614.1। & K00341 & NADH-quinone oxidoreductase subunit L \\
\hline gil188577690|ref|YP_001914619.1। & K00336 & $\mathrm{NADH}$-quinone oxidoreductase subunit $\mathrm{G}$ \\
\hline gil188577693|ref|YP_001914622.1। & K00333 & NADH-quinone oxidoreductase subunit D \\
\hline gil188577770|reflYP_001914699.1। & K03568 & TldD protein \\
\hline gil188578034|ref|YP_001914963.1। & K02014 & Iron complex outermembrane recepter protein \\
\hline gil188578050|reflYP_001914979.1। & K00798 & Cob(I)alamin adenosyltransferase \\
\hline gil188578410|ref|YP_001915339.1l & K02047 & Sulfate transport system permease protein \\
\hline gil188578579|ref|YP_001915508.1। & K02014 & Iron complex outermembrane recepter protein \\
\hline gil188578592|ref|YP_001915521.1। & K02455 & General secretion pathway protein $\mathrm{F}$ \\
\hline gil188578888|ref|YP_001915817.1l & K03497 & Chromosome partitioning protein, ParB family \\
\hline gil188579158|ref|YP_001916087.1| & K01198 & Xylan 1,4-beta-xylosidase \\
\hline gil188579258|ref|YP_001916187.1l & - & - \\
\hline gil229358035|ref|YP_001915183.2| & K02909 & Large subunit ribosomal protein L31 \\
\hline
\end{tabular}


Table 4 Pathway mapping of all essential potential antimicrobial targets

\begin{tabular}{ll}
\hline Pathways & No. of protein \\
\hline Citrate cycle (TCA cycle) & 1 \\
Oxidative phosphorylation & 3 \\
Alanine, aspartate and glutamate metabolism & 1 \\
Arginine and proline metabolism & 1 \\
Glutathione metabolism & 1 \\
Starch and sucrose metabolism & 1 \\
Amino sugar and nucleotide sugar metabolism & 1 \\
Lipopolysaccharide biosynthesis & 1 \\
Carbon fixation pathways in prokaryotes & 1 \\
Porphyrin and chlorophyll metabolism & 1 \\
Nitrogen metabolism & 3 \\
ABC transporters & 1 \\
Two-component system & 2 \\
Bacterial chemotaxis & 1 \\
Ribosome & 1 \\
Protein export & 1 \\
Bacterial secretion system & 2 \\
Cell cycle-Caulobacter & 1 \\
Peroxisome & 1 \\
\hline
\end{tabular}

one each in bacterial chemotaxis and protein export besides several others that are involved in different essential pathways (Table 4). Some of these proteins directly contribute to the basic primary metabolic mechanisms like carbon fixation, phosphorylation, amino acid biosynthesis, citric acid cycle, nitrogen metabolism, etc. However, certain essential proteins like those encoding $\mathrm{ABC}$ transporters (1), bacterial chemotaxis (1), protein export (1) and secretion systems (2) found in the pathogen are, in one or the other way, linked to the pathogenicity, virulence factor, nutrient mobilization and uptake and motility of the organisms (Maranhão et al. 2009; Rodriguez and Smith 2006; Stergiopoulos et al. 2003). Virulent/nonvirulent properties predicted through support vector machine (SVM) approach revealed three virulent proteins (accession number: YP_001911579.1, YP_001913450.1, YP_001914963.1) leading to the assumption that these three essential proteins could have important role in the normal functioning of the pathogen within the host. Thus, these proteins can be viewed as novel targets because of their non-significant similarity with DrugBank targets and can be used for the development of antimicrobials. These three proteins [YP_001911579.1 (TonB-dependent receptor), YP_001913450.1 (Transposase) and YP_001914963.1 (TonB-dependent outer membrane receptor)] are very critical due to their presence in the outer membrane of the pathogens.

\section{Experimental procedure}

Data retrieval

Complete protein sequences of $X$. oryzae pv. oryzae PXO99A and Oryza sativa were downloaded from NCBI (ftp://ftp.ncbi.nih.gov/genomes/). Essential genes of prokaryotes were downloaded from the DEG (http://tubic. tju.edu.cn/deg/) (Zhang et al. 2004). For comparative analysis, known protein targets existing with the DrugBank were downloaded (Wishart et al. 2008).

Identification of essential proteins

Complete protein sequence of $X$. oryzae pv. oryzae PXO99A was subjected to BLASTP (Altschul et al. 1990) against $O$. sativa protein sequences to identify gene products of the pathogen. Sequences that did not show any similarity were further subjected to BLASTP with e-value cutoff score of $10^{-10}$ against all prokaryotic sequences of the DEG (Zhang et al. 2004) to screen out genes that appeared to represent essential genes. Further, biological processes, molecular functions and cellular components have been identified. Complete BLAST alignments were two-way.

Metabolic pathway analysis

Metabolic pathway analysis was carried out by KAAS (KEGG Automatic Annotation Server) at KEGG for the

Xanthomonas oryzae pv. oryzae PXO99A proteome

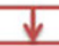

BLAST against rice proteome to select non-homologous proteins (i.e. "No hits found)

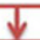

BLAST non-homologous proteins against DEG proteins

Classification of proteins which could be potential targets

Prediction of virulent protein with VirulentPred tool

BLAST virulent protein with DrugBank target for identification of novel target

Fig. 1 Flow chart of systematic identification of novel targets in Xanthomonas oryzae pv. oryzae PXO99A 
identification of essential proteins in different pathways. KAAS provides functional annotation of genes by BLAST comparisons against the manually curated KEGG GENES database. The result contains $\mathrm{KO}$ assignments and automatically generated KEGG pathways (Moriya et al. 2007). The method for identification of probable antibacterial targets is described in Fig. 1.

\section{Prediction of virulent proteins}

Bacterial virulent protein sequences were predicted through bi-layer cascade support vector machine (SVM) based prediction tool VirulentPred (Garg and Gupta 2008). In the first layer SVM classifiers were trained and optimized with different individual protein sequence features and cascaded to the second layer SVM classifier to train and generate the final classifier. The selected prediction approach for the query were amino acid composition, dipeptide composition, similarity searching, higher order dipeptide composition, PSSM and cascased SVM module.

\section{Prediction of targets as novel}

All protein targets of DrugBank (Wishart et al. 2008) were downloaded and aligned with essential proteins for finding out the significant similarity and dissimilarity that represent non-novel and novel targets, respectively. Sequences were subjected to BLASTP with e-value cutoff score of $10^{-4}$ and similarity $>70$ against all the experimental drug targets.

Acknowledgments Financial support from Indian Council of Agricultural Research, India in the form of "National Agricultural Innovation Project" (NAIP) entitled "Establishment of National Agricultural Bioinformatics Grid" (NABG) is gratefully acknowledged.

Conflict of interest Authors declared no conflict of interest.

Open Access This article is distributed under the terms of the Creative Commons Attribution License which permits any use, distribution, and reproduction in any medium, provided the original author(s) and the source are credited.

\section{References}

Altschul SF, Gish W, Miller W, Myers EW, Lipman DJ (1990) Basic local alignment search tool. J Mol Biol 215:403-410

Amineni U, Pradhan D, Marisetty H (2010) In silico identification of common putative drug targets in Leptospira interrogans. J Chem Biol 3(4):165-173

Barh D, Tiwari S, Jain N, Ali A, Santos AR, Misra AN, Azevedo V, Kumar A (2011) In silico subtractive genomics for target identification in human bacterial pathogens. Drug Dev Res 72:162-177

Buysse JM (2001) The role of genomics in antibacterial target discovery. Curr Med Chem 14:1713-1726

Dutta A, Singh SK, Ghosh P, Mukherjee R, Mitter S, Bandyopadhyay D (2006) In silico identification of potential therapeutic targets in the human pathogen Helicobacter pylori. In Silico Biol 6:43-47
Garg A, Gupta D (2008) VirulentPred: a SVM based prediction method for virulent proteins in bacterial pathogens. BMC Bioinform 9:62

Jacobs MA, Alwood A, Thaipisuttikul I, Spencer D, Haugen E, Ernst S, Will O, Kaul R, Raymond C, Levy R, Chun-Rong L, Guenthner D, Bovee D, Olsen MV, Manoil C (2003) Comprehensive transposon mutant library of Pseudomonas aeruginosa. Proc Nat Acad USA 100:14339-14344

Koteswara RG, Nagamalleswara RK, Phani RK, Aravind S (2010) In Silico identification of potential therapeutic targets in Clostridium botulinum by the approach subtractive genomics. Indian $\mathbf{J}$ Bioinform Res 2:12-16

Maranhão FC, Paião FG, Fachin AL, Martinez-Rossi NM (2009) Membrane transporter proteins are involved in Trichophyton rubrum pathogenesis. J Med Microbiol 58:163-168

Miesel L, Greene J, Black TA (2003) Genetic strategies for antibacterial drug discovery. Nat Rev Genet 4:442-456

Moriya Y, Itoh M, Okuda S, Yoshizawa AC, Kanehisa M (2007) KAAS: an automatic genome annotation and pathway reconstruction server. Nucleic Acids Res 35:W182-W185

Niño-Liu DO, Ronald PC, Bogdanove AJ (2006) Xanthomonas oryzae pathovars: model pathogens of a model crop. Mol. Plant Pathol 7(5):303-324

Rice Knowledge Bank (2009) (http://www.knowledgebank.irri.org/ RiceDoctor/information-sheets-mainmenu-2730/diseases-main menu-2735/bacterial-leaf-blight-mainmenu-2758.html)

Rodriguez GM, Smith I (2006) Identification of an ABC transporter required for iron acquisition and virulence in Mycobacterium tuberculosis. J Bacteriol 188:424-430

Sakharkar KR, Sakharkar MK, Chow VT (2004) A Novel Genomics Approach for the Identification of Drug Targets in Pathogens, with Special Reference to Pseudomonas aeruginosa. Silico Biol 4:355-360

Salzberg SL, Sommer DD, Schatz MC, Phillippy AM, Rabinowicz PD, Tsuge S, Furutani A, Ochiai H, Delcher AL, Kelley D, Madupu R, Puiu D, Radune D, Shumway M, Trapnell C, Aparna G, Jha G, Pandey A, Patil PB, Ishihara H, Meyer DF, Szurek B, Verdier V, Koebnik R, Dow JM, Ryan RP, Hirata H, Tsuyumu S, Won Lee S, Seo YS, Sriariyanum M, Ronald PC, Sonti RV, Van Sluys MA, Leach JE, White FF, Bogdanove AJ (2008) Genome sequence and rapid evolution of the rice pathogen Xanthomonas oryzae pv. oryzae PXO99A. BMC Genomics 9:204 (erratum 9: 534)

Stergiopoulos I, Zwiers LH, De Waard MA (2003) The ABC transporter MgAtr4 is a virulence factor of Mycosphaerella graminicola that affects colonization of substomatal cavities in wheat leaves. Mol Plant Microbe Interact 16:689-698

Swings J, Mooter MVD, Vauterin L, Hoste B, Gills M, Mew TW, Kersters K (1990) Reclassification of the causal agents of bacterial blight (Xanthomonas camprestris pv.oryzae) and bacterial leaf streak (Xanthomonas camprestris pv.oryzicola) of rice as pathovars of Xanthomonas oryzae (ex Ishiyama 1922) sp. nov., nom. Rev Int J Syst Bacteriol 40:309-311

Triplett LR, Hamilton JP, Buell CR, Tisserat NA, Verdier V, Zink F, Leach JE (2011) Genomic analysis of Xanthomonas oryzae isolates from rice grown in the United States reveals substantial divergence from known $X$. oryzae pathovars. Appl Environ Microbiol 77:3930-3937

Vetrivel U, Subramanian G, Dorairaj S (2011) A novel in silico approach to identify potential therapeutic targets in human bacterial pathogens. Hugo J 5(1-4):25-34

Wishart DS, Knox C, Guo AC, Cheng D, Shrivastava S, Tzur D, Gautam B, Hassanali M (2008) DrugBank: a knowledgebase for drugs, drug actions and drug targets. Nucleic Acids Res 36(Database issue):D901-D906

Zhang R, Ou HY, Zhang CT (2004) DEG: a database of essential genes. Nucleic Acids Res 32:D271-D272 